\title{
Amlodipine or lisinopril was not better than chlorthalidone in lowering CHD risk in hypertension
}

\begin{abstract}
Major outcomes in high-risk hypertensive patients randomized to angiotensin-converting enzyme inhibitor or calcium channel blocker vs diuretic: The Antihypertensive and Lipid-Lowering Treatment to Prevent Heart Attack Trial (ALLHAT).JAMA 2002;288:2981-97.

\section{QUESTION: In predominately older patients with hypertension and $\geq 1$ other coronary heart disease (CHD) risk factor, is amlodipine or lisinopril better than chlorthalidone in lowering the risk for CHD or other cardiovascular disease (CVD) events?}

\section{Design}

Randomised (allocation concealed*), blinded \{patients, clinicians, data collectors, outcome assessors, and steering committee $\} \dagger^{*}{ }^{*}$ controlled trial with mean 4.9 years of follow up (Antihypertensive and LipidLowering Treatment to Prevent Heart Attack Trial [ALLHAT]).

\section{Setting}

623 centres in the US, Canada, Puerto Rico, and the US Virgin Islands.

\section{Patients}

33357 patients $\geq 55$ years of age (mean age 67 y, $53 \%$ men) who had stage 1 or stage 2 hypertension and $\geq 1$ additional risk factor for CHD. The risk factors were myocardial infarction (MI) or stroke in the previous 6 months, left ventricular hypertrophy, type 2 diabetes, smoking, high density lipoprotein cholesterol $<0.91$ $\mathrm{mmol} / \mathrm{l}$, or other atherosclerotic CVD. Patients with a history of heart failure (HF) or known left ventricular ejection fraction $<35 \%$ were excluded. Follow up was 97\%; all randomised patients were included in the analysis.

\section{Intervention}

Patients were allocated to chlorthalidone, 12.5-25 $\mathrm{mg} /$ day $\quad(\mathrm{n}=15$ 255); amlodipine, 2.5-10 $\mathrm{mg}$ /day ( $\mathrm{n}=9048)$; or lisinopril, $10-40 \mathrm{mg} /$ day $(\mathrm{n}=9054)$.

\section{Main outcome measures}

Combined fatal CHD or nonfatal MI. Secondary outcomes were all cause mortality, fatal and nonfatal stroke, combined CHD (the primary outcome, coronary revascularisation [coronary artery bypass graft, percutaneous angioplasty, insertion of stents, and atherectomy], and hospital admission for angina), and combined CVD (combined CHD, stroke, other treated angina, HF [fatal, hospitalised, or treated non-hospitalised], and peripheral arterial disease).

\section{Main results}

Analysis was by intention to treat. Patients who received amlodipine or lisinopril did not differ from those who received chlorthalidone for the primary outcome (table). The amlodipine group had a 38\% (95\% CI 25 to 52) relative risk increase for $\mathrm{HF}$ and a $35 \%$ (CI 21 to 50) greater risk of hospitalised fatal $\mathrm{HF}$ than did the chlorthalidone group. The lisinopril group had a 15\% (CI 2 to 30) greater risk of stroke and a 10\% (CI 5 to 16) greater risk of combined CVD than did the chlorthalidone group.

\section{Conclusions}

In older patients with hypertension and $\geq 1$ other coronary heart disease (CHD) risk factor, amlodipine or lisinopril was no better than chlorthalidone in lowering the risk of CHD or other cardiovascular disease (CVD) events. Risks of some CVD events were greater with amlodipine or lisinopril.

*See glossary.

$\dagger$ Information provided by author.
Sources of funding: National Heart, Lung, and Blood Institute and Pfizer.

For correspondence: Dr J $T$ Wright, Case Western Reserve University, Cleveland, $\mathrm{OH}, \mathrm{USA}$.

jxw20@po.cwru.edu

Dr B R Davis,

University of

Texas-Houston Health

Science Center,

Houston, TX, USA.

bdavis@sph.uth.tmc.edu

Abstracts and

commentary also

appear in ACP Journal Club.

Amlodipine or lisinopril $v$ chlorthalidone for combined fatal CHD or non-fatal MI at mean 4.9 years

\begin{tabular}{lllll} 
Amlodipine & Lisinopril & Chlorthalidone & RRR $(95 \%$ CI) & NNT \\
$11.3 \%$ & - & $11.5 \%$ & $2 \%(-7$ to 10$)$ & Not significant \\
\hline- & $11.4 \%$ & $11.5 \%$ & $1 \%(-8$ to 9$)$ & Not significant \\
\hline
\end{tabular}

$\ddagger \mathrm{CHD}=$ coronary heart disease; $\mathrm{Ml}=$ myocardial infarction. Other abbreviations defined in glossary .

\section{COMMENTARY}

A study of astonishing methodological beauty, ALLHAT is a large, simple, practice based, double blind, active comparison trial that poses important public health questions about the effects of first line antihypertensive therapies on major health outcomes, including MI, stroke, and HF. This combination of excellent methods and outstanding questions means that the findings merit careful attention, wide dissemination, and clinical translation.

In ALLHAT, neither the calcium channel blocker amlodipine nor the ACE inhibitor lisinopril was better than the low dose diuretic chlorthalidone for any primary or secondary outcome. Conversely, low dose diuretics were superior to amlodipine or lisinopril for at least 1 major disease outcome. Low dose diuretics alone should be the first line drug therapy for hypertension.

For patients starting drug therapy, low dose diuretics should be the initial therapy for uncomplicated hypertension, although it may take 4 weeks to achieve maximum effect. If blood pressure (BP) is not controlled on diuretics alone, a second drug should be added. Many patients are already treated, but not with a diuretic. For those with uncontrolled BP on a non-diuretic regimen, a low dose diuretic should be added. If BP is controlled with a non-diuretic, the patient should be switched to a low-dose diuretic.

The ALLHAT trial, led by academic researchers and funded by the US National Institutes of Health and Pfizer, represents an important university industry collaboration: newer drugs were compared with an established drug instead of a placebo for clinically important outcomes. Diuretics should be the standard reference group in future comparative trials.

The superiority of low dose diuretics is not likely to be promoted by industry. Indeed, "opinion leaders" argue that BP control is inadequate; everyone needs 2 drugs, so combination therapy should be first line therapy. ${ }^{1}$ During ALLHAT, which used the traditional stepped care approach, $40-60 \%$ of patients were taking only 1 drug. For the large proportion of patients with hypertension whose BP can be controlled on 1 drug, the 2 drugs for everyone approach, which remains largely untested, would unnecessarily expose many patients to adverse effects without known benefit.

Bruce M Psaty, MD, PhD University of Washington, Seattle, Washington, USA

1 Opie LH, Messerli FH. The choice of first-line therapy: rationale for low-dose combinations of an angiotensin converting enzyme inhibitor and a diuretic. J Hypertens Suppl.
2001;19:S17-21. 